\title{
ESTUDO LONGITUDINAL DE INDIVÍDUOS COM DOENÇA DE CHAGAS DE REGIÃO ENDÊMICA BRASILEIRA: A COORTE SAMI- TROP
}

\section{Longitudinal Study of Individuals with Chagas Disease from Brazilian Endemic Region: the Sami-Trop Cohort}

\author{
Andréia Brito de Souza ${ }^{1}$ \\ Amanda Mota Lacerda ${ }^{2}$ \\ Amanda Karoline Pinheiro Silva ${ }^{3}$ \\ Ariela Mota Ferreira ${ }^{4}$ \\ Renata Fiúza Damasceno ${ }^{5}$ \\ Éster Cerdeira Sabino ${ }^{6}$ \\ Antônio Luiz Pinho Ribeiro ${ }^{7}$ \\ Thallyta Maria Vieira ${ }^{8}$ \\ Desirée Sant'Ana Haikal ${ }^{9}$
}

\footnotetext{
${ }^{1}$ Mestre em Ciências da Saúde Universidade Estadual de Montes Claros (UNIMONTES). Montes, Claros MGBrasil. $\square$ brito.ad2014@gmail.com. (I) https://orcid.org/0000-0002-3708-3765.

${ }^{2}$ Mestre em Ciências da Saúde Universidade Estadual de Montes Claros (UNIMONTES). Montes, Claros MGBrasil. $\square$ amandamlacerda@yahoo.com.br. (D) https://orcid.org/0000-0001-5333-4686).

${ }^{3}$ Graduada em Enfermagem. Universidade Estadual de Montes Claros (UNIMONTES). Montes Claros, MGBrasil. $\square$ karolinepinheiro25@gmail.com. (1) https://orcid.org/0000-0002-1060-3062.

${ }^{4}$ Mestre em Ciências da Saúde Universidade Estadual de Montes Claros (UNIMONTES). Doutoranda em Ciências da Saúde Universidade Estadual de Montes Claros (UNIMONTES). Montes Claros, MG- Brasil. $\square$ arielamota@ hotmail.com. (I) https://orcid.org/0000-0002-2315-5318.

${ }^{5}$ Mestre em Ciências da Saúde Universidade Estadual de Montes Claros (UNIMONTES). Doutoranda em Ciências da Saúde Universidade Estadual de Montes Claros (UNIMONTES). Montes Claros, MG- Brasil. $\square$ damascenoenf@gmail.com. (1) https://orcid.org/0000-0002-9525-8527.

${ }^{6}$ Doutora em Imunologia. Universidade de São Paulo (USP), São Paulo, SP-Brasil. Instituto da Universidade de São Paulo. São Paulo, SP- Brasil. $\$ sabinoec@ gmail.com. (1) https://orcid.org/0000-0003-2623-5126.

7 Doutor em Infectologia e Medicina Tropical. Universidade Federal de Minas Gerais (UFMG), Brasil. Departamento de Clínica Médica. Belo Horizonte, MG- Brasil. $\square$ tom1963br@yahoo.com.br. https://orcid.org/0000-0002-2740-0042.

${ }^{8}$ Doutora em Parasitologia. Universidade Federal de Minas Gerais (UFMG). Belo Horizonte, MG- Basil. Programa de Pós-Graduação em Ciências da Saúde, Universidade Estadual de Montes Claros (UNIMONTES). Montes Claros, MG- Brasil. $\square$ thallytabio@gmail.com. (D) https://orcid.org/0000-0002-1483-6234.

${ }^{9}$ Doutora em Odontologia em Saúde Coletiva. Faculdade de Odontologia da Universidade Federal de Minas Gerais (UFMG), Belo Horizonte, MG -Brasil. Programa de Pós-Graduação em Ciências da Saúde, Universidade Estadual de Montes Claros (UNIMONTES). Montes Claros, MG- Brasil. $\square$ desireehaikal@gmail.com. https://orcid.org/0000-0002-0331-0747.
}

$\begin{array}{ccc}\text { Recebido em } & \text { Aceito em } & \text { Publicado em } \\ 15 / 03 / 2021 & 03 / 07 / 2021 & 27 / 07 / 2021\end{array}$


Resumo: a coorte SaMi-Trop - Centro de Pesquisa em Medicina Tropical de São PauloMinas Gerais, um estudo multicêntrico de pacientes com Doença de Chagas - DC, idealizado por cientistas de quatro universidades públicas brasileiras, tem sido conduzida desde $2013 \mathrm{em}$ regiões endêmicas do estado de Minas Gerais - MG. Objetivo: apresentar e descrever como está sendo conduzida a coorte SaMi-Trop e seus principais resultados, com um retorno local voltado aos gestores municipais. Métodos: os participantes foram recrutados através da base de dados da Rede de Teleassistência de Minas Gerais - RTMG. Até o momento, duas coletas foram realizadas, uma linha de base que ocorreu nos anos de 2013 e 2014 e um primeiro seguimento nos anos de 2015 a 2016. Resultados: participaram 2157 indivíduos na linha de base. Desses, 146 faleceram e 1709 se mantiveram no primeiro seguimento, após dois anos. A maioria dos participantes era do sexo feminino, apresentava idade até 60 anos, união estável e se autodeclararam não branco. Conclusão: a coorte tem contribuído com o avanço no conhecimento científico da DC. Seus resultados, também, devem subsidiar políticas públicas e estratégias para melhorar os serviços de saúde ofertados, de forma direcionada, às reais necessidades dos portadores de DC.

Palavras-chave: Doença de Chagas; Doenças Negligenciadas; Cardiomiopatia Chagásica.

Abstract: the SaMi-Trop cohort (Center for Research in Tropical Medicine of São PauloMinas Gerais), a multicentric study of patients with Chagas' disease (CD) designed by scientists from four Brazilian public universities, has been conducted since 2013 in regions endemic to the state of Minas Gerais (MG). Objective: to present and describe how the SaMiTrop cohort and its main results are being conducted, also promoting a local return aimed at municipal managers. Methods: participants were recruited through the database of the Minas Gerais Telecare Network (RTMG). To date, two collections have been made, the baseline that took place in the years 2013 and 2014 and a first follow-up in the years 2015 to 2016. Results: 2157 individuals participated in the baseline. Of these, 146 died and 1709 remained in the first follow-up, two years later. Most participants were female, aged up to 60 years, had a stable union and declared themselves to be non-white. Conclusion: the cohort has contributed to the advancement in scientific knowledge of CD. Its results should also support public policies and strategies to improve the health services offered in a way that addresses the real needs of people with CD.

Keywords: Chagas Disease; Neglected Diseases; Chagas Cardiomyopathy.

\section{INTRODUÇÃO}

A Doença de Chagas - DC é uma infecção causada pelo protozoário Trypanossoma cruzi, sendo considerada uma doença negligenciada pela Organização Mundial da Saúde - OMS ${ }^{1}$. Está relacionada com importante morbidade e mortalidade em pessoas de idade ativa, podendo provocar danos prevalentes a populações desfavorecidas ${ }^{2}$. A DC possui uma fase aguda, que acontece após a transmissão inicial, em que o indivíduo manifesta sintomas inespecíficos, dificultando o diagnóstico ${ }^{3}$. Cerca de 60 a $70 \%$ dos portadores de DC 
se encontram na forma crônica indeterminada, condição com ausência de sinais cardíacos e digestivos ${ }^{4}$. De 10 a 30 anos, após a infecção primária, 30-40\% dos indivíduos podem evoluir para Cardiomiopatia Chagásica Crônica - CCC, manifestação mais constante e crítica da DC, que pode levar a insuficiência cardíaca, sendo esta a principal causa de morte entre portadores da $\mathrm{DC}^{5}$, representando $38 \%$ da mortalidade de pacientes com doença cardíaca ${ }^{6}$.

A OMS estima que há cerca de oito milhões de pessoas contaminadas por DC no mundo, com uma mortalidade anual de 10 mil pessoas, devido a complicações da doença ${ }^{7}$. Grande parte desses casos está na América Latina, onde o Brasil é o país com maior número de pessoas contaminadas (aproximadamente 4,6 milhões de indivíduos). Entre os anos de 2009 a 2018 foram registrados 45.863 óbitos, devido a DC. Destes, 11.343 (24,7\%) dos óbitos aconteceram no estado de Minas Gerais - MG, com destaque para a macrorregião Norte que apresentou 2.300 óbitos (20,28\%), sendo esta a maior taxa de mortalidade do estado no período citado, caracterizando esta macrorregião como endêmica ${ }^{8,9}$.

Com o objetivo de contribuir para o conhecimento desta doença, uma grande coorte de pacientes com DC tem sido conduzida, desde 2013, em regiões endêmicas do estado de Minas Gerais - MG. Trata-se da coorte SaMi-Trop - Centro de Pesquisa em Medicina Tropical de São Paulo-Minas Gerais, um estudo multicêntrico idealizado por cientistas de quatro universidades públicas brasileiras dos estados de Minas Gerais - MG e São Paulo $\mathrm{SP}^{10}$. A coorte tem como objetivo identificar biomarcadores e outros preditores para risco de progressão e de morte pela $\mathrm{DC}^{11}$. A coorte vem acompanhando cerca de 2000 indivíduos com DC e já tem apresentado resultados significativos ${ }^{11-13}$. Assim, o estudo evidenciado, aqui, apresenta e descreve como está sendo conduzida a coorte SaMi-Trop e seus principais resultados, promovendo um retorno local, voltado aos gestores municipais.

\section{DESCRIÇÃO DA COORTE SAMI-TROP}

O SaMi-Trop é um estudo de coorte multicêntrico de portadores de DC residentes em 21 municípios do estado de Minas Gerais - MG, com acompanhamento desde 2013. O estudo é fruto de parceria entre quatro universidades públicas brasileiras: Universidade de São Paulo - USP, Universidade Estadual de Montes Claros - UNIMONTES, Universidade Federal de Minas Gerais - UFMG e Universidade Federal de São João del-Rei - UFSJ. Possui 
financiamento do National Institutes of Health - NIH dos Estados Unidos (grant number 1P50AI098461).

\section{Recrutamento de participantes}

Os participantes foram recrutados através da base de dados da Rede de Teleassistência de Minas Gerais - RTMG, um programa que fornece assistência à distância para unidades públicas de saúde com apoio remoto em laudos de exames ${ }^{14}$. Este recrutamento teve como base exames de eletrocardiograma - ECG, realizados pela RTMG nos anos de 2011 e $20^{12}$, considerando munícipios pertencentes a duas mesorregiões endêmicas para DC do estado de Minas Gerais - MG: a mesorregião do Norte e a mesorregião do Vale do Jequitinhonha. Foram selecionados participantes que atendiam aos seguintes critérios: idade superior a 18 anos; autorrelato de DC e apresentar alterações no ECG (possível infarto do miocárdio antigo, uso de marcapasso ou outras alterações características da DC). Os critérios de exclusão do estudo foram: gestantes, lactantes e portadores de doença com risco de vida. A partir da identificação dos indivíduos elegíveis, foram selecionados os municípios, com base em sua localização e o número de casos apresentados da DC.

Inicialmente, os gestores de saúde de cada município foram contatados e convidados a participarem do estudo. Os gestores indicaram um profissional da Atenção Primária à Saúde - APS, para contribuir na captação de participantes e agendamento da coleta de dados. Esses profissionais da APS, de cada um dos 21 municípios, foram treinados, calibrados, fizeram um curso de ética, ofertado pela equipe SaMi-Trop e assinaram um termo de sigilo para manuseio de dados relacionados ao projeto.

\section{Estratégia de coleta de dados e condições avaliadas}

Até o momento, duas coletas foram realizadas, a linha de base que ocorreu nos anos de 2013 e 2014, e, posteriormente, um primeiro seguimento nos anos de 2015 a 2016. Um novo seguimento de avaliação está em andamento, tendo sido iniciado no ano de 2019 (dados não mostrados). As coletas de dados foram realizadas nas Unidades Básicas de Saúde - UBS, onde os participantes foram entrevistados e examinados por pesquisadores treinados para tais fins. Os dados foram registrados em formulário eletrônico padronizado e as informações enviadas para base de dados centralizada no Instituto de Medicina Tropical da Universidade de São Paulo - IMT/USP. As temáticas abordadas na entrevista da linha de base 
e do segmento são: informações sociodemográficas, hábitos de vida, história clínica, tratamento da DC, atividade física, qualidade de vida, e, no seguimento, também, foram incluídas: utilização dos serviços de saúde, alfabetização em saúde, hospitalização nos últimos dois anos.

$\mathrm{Na}$ linha de base, além da entrevista, foram realizados amostras de sangue e exames de ECG. Os participantes foram todos submetidos a exames de sorologia para confirmação da DC e, aqueles que apresentaram resultados negativos, foram submetidos a dois diferentes métodos de sorologia para esta confirmação. No primeiro seguimento, realizado dois anos após a linha de base, além de todos os procedimentos conduzidos nessa linha de base, a entrevista foi ampliada e houve a realização do exame de ecocardiograma ECO.

Todos os profissionais envolvidos na coleta de dados possuíam graduação na área da saúde e passaram por treinamento e coleta de dados. As amostras de sangue foram enviadas ao laboratório central do IMT/USP. Os exames de ECG foram encaminhados eletronicamente a RTMG, quando foram analisados e laudados por cardiologistas. A aquisição de imagens do ECO foi realizada por um enfermeiro, devidamente treinado, e o laudo foi realizado de forma remota por cardiologistas da equipe do SaMi-Trop.

\section{Aprovação ética}

O projeto SaMi-Trop recebeu aprovação ética do Comitê de Ética em Pesquisa da Faculdade de Medicina da Universidade de São Paulo, número do protocolo: 042/2012; do Conselho Nacional de Revisão Institucional - CONEP, número: 179.685 / 2012); e do Comitê de Ética em Pesquisa da Universidade Estadual de Montes Claros, número do parecer: 2.474.172. Todos os sujeitos concordaram em participar deste estudo e assinaram o termo de consentimento informado antes de iniciar a coleta o estudo.

\section{ANÁLISES CONDUZIDAS NESTE ESTUDO}

Esse estudo avaliou descritivamente algumas informações coletadas na linha de base e no primeiro seguimento da coorte SaMi-Trop. As informações analisadas, na linha de base, foram agrupadas em características sociodemográficas, histórico médico e comportamento em saúde. No primeiro seguimento, as informações analisadas foram agrupadas em perda de seguimento, agravamento da doença e serviço de saúde (Quadro 1). 
Quadro 1.Variáveis contempladas neste estudo, segundo perguntas realizadas, opções de resposta, categorização adotada e seguimento em que foram coletadas. Coorte SaMi-Trop. Minas Gerais - MG - Brasil.

\begin{tabular}{|c|c|c|c|c|c|}
\hline \multirow{12}{*}{ 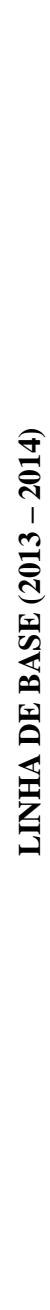 } & \multirow{7}{*}{ 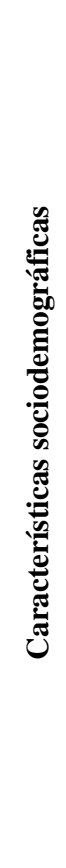 } & & Forma de aferição & Opções de respostas & Categorização adotada \\
\hline & & Sexo & Sexo participante ${ }^{x}$ & $\begin{array}{l}\text { Masculino/ } \\
\text { Feminino }\end{array}$ & $\begin{array}{l}\text { Masculino/ } \\
\text { Feminino }\end{array}$ \\
\hline & & Idade & Idade do participante (anos) $)^{x}$ & Informação numérica & $\begin{array}{l}\text { Até } 60 \text { anos/ } \\
60 \text { anos ou mais }\end{array}$ \\
\hline & & Renda percapita & $\begin{array}{l}\text { "Qual a sua renda familiar?" (em reais) dividida pelo } \\
\text { número de moradores da residência }{ }^{x}\end{array}$ & Informação numérica & $\begin{array}{l}\text { Superior à média percapital } \\
\text { Inferior à média per capita* }\end{array}$ \\
\hline & & Estado civil & "Qual o estado civil do(a) senhor (a)?"x & $\begin{array}{l}\text { Casado ou coabita/ } \\
\text { Solteiro/ divorciado/ viúvo }\end{array}$ & $\begin{array}{l}\text { Com união estável/ } \\
\text { Sem união estável }\end{array}$ \\
\hline & & Cor da pele auto-declarada & "Qual a sua cor?" & $\begin{array}{l}\text { Branca/ } \\
\text { Amarela/ indígena/ parda/ preta }\end{array}$ & $\begin{array}{l}\text { Branca/ } \\
\text { Amarela/ indígena/ parda/ preta }\end{array}$ \\
\hline & & Alfabetizado & "O(a) senhor (a) sabe ler e escrever?" & $\begin{array}{l}\text { Não/ } \\
\text { Sim }\end{array}$ & $\begin{array}{l}\text { Não/ } \\
\text { Sim }\end{array}$ \\
\hline & \multirow{5}{*}{ 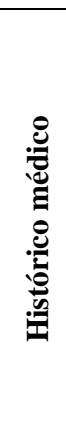 } & Colesterol alto & $\begin{array}{l}\text { "Nos últimos dois anos, algum médico disse que o(a) } \\
\text { senhor(a) estava com o colesterol alto?" }\end{array}$ & $\begin{array}{l}\text { Não/ } \\
\text { Sim }\end{array}$ & $\begin{array}{l}\text { Não/ } \\
\text { Sim }\end{array}$ \\
\hline & & Diabetes mellitus & $\begin{array}{l}\text { "Nos últimos dois anos, algum médico disse que o(a) } \\
\text { senhor(a) estava com Diabetes mellitus?"§ }\end{array}$ & $\begin{array}{l}\text { Não/ } \\
\text { Sim }\end{array}$ & $\begin{array}{l}\text { Não/ } \\
\text { Sim }\end{array}$ \\
\hline & & Hipertensão arterial & $\begin{array}{l}\text { "Nos últimos dois anos, algum médico disse que o(a) } \\
\text { senhor(a) estava com o Hipertensão arterial?" }\end{array}$ & $\begin{array}{l}\text { Não/ } \\
\text { Sim }\end{array}$ & $\begin{array}{l}\text { Não/ } \\
\text { Sim }\end{array}$ \\
\hline & & DC na família & $\begin{array}{l}\text { "Além do(a) senhor (a), mais alguém da sua família } \\
\text { (incluindo pais, irmãos ou filhos) tem DC”? }\end{array}$ & $\begin{array}{l}\text { Não/ } \\
\text { Sim }\end{array}$ & $\begin{array}{l}\text { Não/ } \\
\text { Sim }\end{array}$ \\
\hline & & Uso prévio de $\mathrm{BZN}^{\ddagger}$ & $\begin{array}{l}\text { "O(a) senhor (a) já tomou um medicamento chamado } \\
\text { ROCHAGAN ou BENZONIDAZOL"? }\end{array}$ & $\begin{array}{l}\text { Não/ } \\
\text { Sim }\end{array}$ & $\begin{array}{l}\text { Não/ } \\
\text { Sim }\end{array}$ \\
\hline
\end{tabular}




\section{RC}

\begin{tabular}{|c|c|c|c|}
\hline Portador de marca-passo & "O(a) senhor(a) tem marca-passo permanente?"メ & $\begin{array}{l}\text { Não/ } \\
\text { Sim }\end{array}$ & $\begin{array}{l}\text { Não/ } \\
\text { Sim }\end{array}$ \\
\hline Classe Funcional & Avaliação médica & $\begin{array}{l}\text { Classe I } \\
\text { Classe II/III/IV }\end{array}$ & $\begin{array}{l}\text { Sem limitações/ } \\
\text { Com limitações }\end{array}$ \\
\hline NT-proBNP & Exame laboratorial & Medida numérica & $\begin{array}{l}\text { Não alterado }(<300) \\
\text { Alterado }(\geq 300)\end{array}$ \\
\hline $\begin{array}{l}\text { Autopercepção da saúde } \\
\text { Colocar depois de NT- } \\
\text { proBNP }\end{array}$ & $\begin{array}{l}\text { "Como o(a) senhor (a) classificaria sua saúde } \\
\text { hoje?"x }\end{array}$ & $\begin{array}{l}\text { Média/ boa/ muito boa/ } \\
\text { Muito ruim/ ruim }\end{array}$ & $\begin{array}{l}\text { Positiva/ } \\
\text { Negativa }\end{array}$ \\
\hline Qualidade de vida & "Como você avaliaria sua qualidade de vida?"メ & $\begin{array}{l}\text { Muito ruim/ruim/ } \\
\text { Nem ruim nem boa/ } \\
\text { Boa/muito boa }\end{array}$ & $\begin{array}{l}\text { Insatisfatória/ } \\
\text { Regular/ } \\
\text { Satisfatória }\end{array}$ \\
\hline Prática de atividade física & "O(a) senhor(a) pratica alguma atividade fisica?" & $\begin{array}{l}\text { Não/ } \\
\text { Sim }\end{array}$ & $\begin{array}{l}\text { Não/ } \\
\text { Sim }\end{array}$ \\
\hline
\end{tabular}

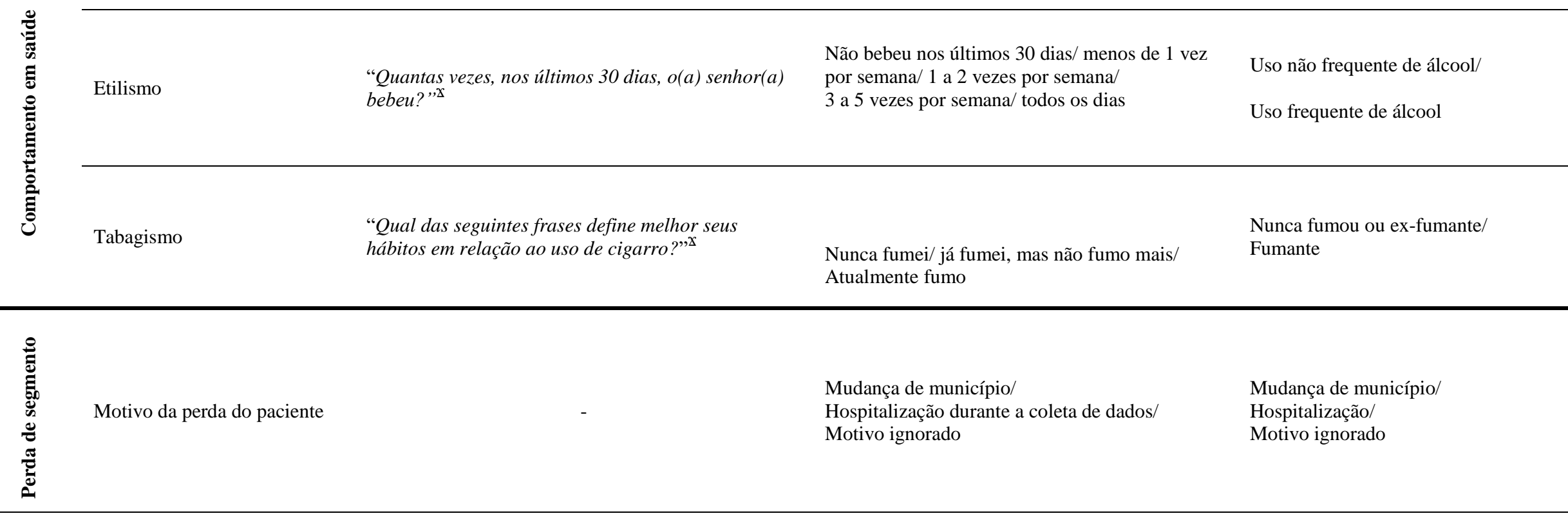


Implantação de marca-passo “ O(a) senhor(a) colocou marca-passo nos últimos $2 \quad$ Não/ Não/

nos últimos dois anos anos?"メ $\quad$ Sim Sim

Surgimento de fibrilação atrial ECG _ _ _ Não/

nos últimos dois anos $\quad$ ECG

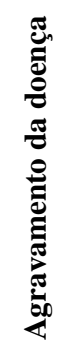

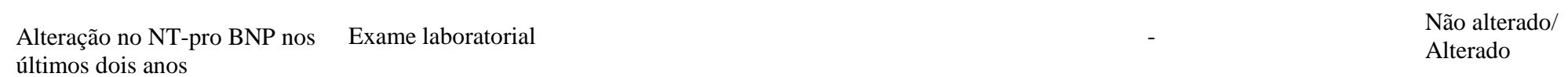

"Voce foi hospitalizado(a) nos ultimos 2 anos?

$\begin{array}{llll}\text { Hospitalização nos últimos } & \text { "Vocêfoi hospitalizado(a) nos últimos } 2 \text { anos?"» } & \text { Não/ } & \text { Não/ } \\ \text { dois anos } & \text { Sim }\end{array}$

$\begin{array}{lll}\text { Óbito } & \begin{array}{l}\text { Perda de seguimento por óbito notificado pelo } \\ \text { município }\end{array} & - \\ \text { Sim }\end{array}$

\begin{tabular}{|c|c|c|c|c|}
\hline \multirow{3}{*}{ 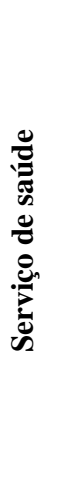 } & Serviço de saúde para DC & $\begin{array}{l}\text { "Qual tipo de serviço de saúde o(a) senhor(a) utiliza } \\
\text { com maior frequência na condução de seu } \\
\text { tratamento da Doença de Chagas?"メ }\end{array}$ & $\begin{array}{l}\text { Público/ } \\
\text { Privado/ Plano de saúde } \\
\text { Nenhum }\end{array}$ & $\begin{array}{l}\text { Público/ } \\
\text { Privado/ Plano de saúde } \\
\text { Nenhum }\end{array}$ \\
\hline & $\begin{array}{l}\text { Residência acompanhada pela } \\
\text { ESF }^{\epsilon}\end{array}$ & $\begin{array}{l}\text { "Sua residência está localizada em região } \\
\text { acompanhada pelo ESF?"x }\end{array}$ & $\begin{array}{l}\text { Não/ } \\
\text { Sim/ } \\
\text { Não sabe }\end{array}$ & $\begin{array}{l}\text { Não/ } \\
\text { Sim/ } \\
\text { Não sabe }\end{array}$ \\
\hline & $\begin{array}{l}\text { Acompanhamento pelo } \\
\text { médico da ESF na condução } \\
\text { da DC }\end{array}$ & $\begin{array}{l}\text { "O(a) senhor (a) é acompanhado(a), de forma } \\
\text { regular ou periódica, por um médico da ESF para o } \\
\text { tratamento da DC?"§ }\end{array}$ & $\begin{array}{l}\text { Sem acompanhamento/ } \\
\text { Irregular/ } \\
\text { Regular/ } \\
\text { Não sabe }\end{array}$ & $\begin{array}{l}\text { Sem acompanhamento/ } \\
\text { Irregular/ } \\
\text { Regular/ } \\
\text { Não sabe }\end{array}$ \\
\hline
\end{tabular}




\begin{tabular}{|c|c|c|c|}
\hline $\begin{array}{l}\text { Acompanhamento por médico } \\
\text { especialista na condução da } \\
\text { DC }\end{array}$ & $\begin{array}{l}\text { "O(a) senhor (a) é acompanhado(a), de forma } \\
\text { regular ou periódica, por um médico especialista } \\
\text { para o tratamento da DC?" }\end{array}$ & $\begin{array}{l}\text { Sem acompanhamento/ } \\
\text { Irregular/ } \\
\text { Regular/ } \\
\text { Não sabe }\end{array}$ & $\begin{array}{l}\text { Sem acompanhamento/ } \\
\text { Irregular/ } \\
\text { Regular/ } \\
\text { Não sabe }\end{array}$ \\
\hline $\begin{array}{l}\text { Satisfação com o tratamento } \\
\text { para DC }\end{array}$ & $\begin{array}{l}\text { "O(a) senhor(a) se sente satisfeito(a) com o } \\
\text { atendimento/tratamento que está recebendo do } \\
\text { serviço público de saúde com relação à DC?"» }\end{array}$ & $\begin{array}{l}\text { Satisfeito/razoavelmente satisfeito/ } \\
\text { Insatisfeito/muito insatisfeito/ } \\
\text { Não sabe }\end{array}$ & $\begin{array}{l}\text { Satisfeito/razoavelmente satisfeito/ } \\
\text { Insatisfeito/muito insatisfeito/ } \\
\text { Não sabe }\end{array}$ \\
\hline $\begin{array}{l}\text { Tempo desde a última } \\
\text { consulta para DC }\end{array}$ & $\begin{array}{l}\text { "Qual o tempo decorrido desde sua última consulta } \\
\text { médica relacionada a DC?"§ }\end{array}$ & Numérica & $\begin{array}{l}\text { Menos de um ano/ } \\
\text { Mais de um ano }\end{array}$ \\
\hline Distância da residência à ESF & $\begin{array}{l}\text { "A ESF, onde o(a) senhor(a) é atendido(a) com } \\
\text { maior frequência, está localizada à } \\
\text { aproximadamente quantos quilômetros de sua } \\
\text { residência?"» }\end{array}$ & Numérica & $\begin{array}{l}\text { Até } 5 \mathrm{~km} / \\
\text { De } 6 \text { a } 99 \mathrm{~km} / \\
\text { Acima de } 100 \mathrm{~km}\end{array}$ \\
\hline $\begin{array}{l}\text { Compreensão da condição de } \\
\text { saúde para DC }\end{array}$ & $\begin{array}{l}\text { "O(a) senhor(a) considera que compreende sua } \\
\text { situação de saúde e os cuidados que deve tomar } \\
\text { durante seu tratamento para DC?" }\end{array}$ & $\begin{array}{l}\text { Compreende bem/ razoavelmente/ } \\
\text { Pouca compreensão/ } \\
\text { Não sabe }\end{array}$ & $\begin{array}{l}\text { Compreende bem/ razoavelmente/ } \\
\text { Pouca compreensão/ } \\
\text { Não sabe }\end{array}$ \\
\hline $\begin{array}{l}\text { Medicamento gratuito para } \\
\text { DC }\end{array}$ & $\begin{array}{l}\text { "Com que frequência o(a) senhor (a) consegue obter } \\
\text { gratuitamente do serviço público, os medicamentos } \\
\text { que lhe foram prescritos para o tratamento da } \\
D C ?, " x\end{array}$ & $\begin{array}{l}\text { Sempre/Frequentemente/ } \\
\text { Regularmente/ } \\
\text { Raramente/ Nunca } \\
\text { Não sabe/recusa a responder }\end{array}$ & $\begin{array}{l}\text { Sempre/Frequentemente/ } \\
\text { Regularmente/ } \\
\text { Raramente/ Nunca } \\
\text { Não sabe/recusa a responder }\end{array}$ \\
\hline $\begin{array}{l}\text { Frequências de exames para } \\
\text { DC }\end{array}$ & $\begin{array}{l}\text { "Com que frequência o(a) senhor(a) consegue } \\
\text { realizar os exames solicitados na condução de seu } \\
\text { tratamento para Doença de Chagas?" }\end{array}$ & $\begin{array}{l}\text { Sempre/Frequentemente/ } \\
\text { Regularmente/ } \\
\text { Raramente/ Nunca }\end{array}$ & $\begin{array}{l}\text { Sempre/Frequentemente/ } \\
\text { Regularmente/ } \\
\text { Raramente/ Nunca }\end{array}$ \\
\hline
\end{tabular}

${ }^{x}$ Pergunta contemplada na entrevista. * Renda média per capita de R \$356,32. ${ }^{*}$ BZN: Benzonidazol. ${ }^{€}$ ESF: Estratégia Saúde da Família. 
O software utilizado para as análises estatísticas foi o SPSS $^{\circledR}$ versão 19. Foi realizada a análise descritiva das variáveis da linha de base e do primeiro segmento. Foram estimadas as frequências simples (n) e relativas (\%) para as variáveis categóricas. Para as variáveis numéricas, idade e renda per capta, foram estimadas médias e desvios padrão. A fim de testar a associação entre as variáveis avaliadas na linha de base (variáveis independentes) e a ocorrência de óbito (variável dependente) foi conduzida análise bivariada, através do teste Qui-quadrado de Person. Foi adotado nível de significância de $5 \%(\alpha \leq 0,05)$.

\section{RESULTADOS}

Participaram do estudo 2157 indivíduos na linha de base da coorte SaMi-Trop. Desses, 146 faleceram e 1709 se mantiveram no primeiro seguimento, após dois anos. Houve perda de 302 participantes, dos quais 273 desistiram, 21 haviam mudado de município, 06 não participaram por motivo ignorado e 02 se encontravam hospitalizados na ocasião da coleta de dados.

A maioria dos participantes da linha de base, bem como do primeiro seguimento, pertenciam ao município de São Francisco (Gráfico 1).

Gráfico 1 - Distribuição dos participantes da linha de base $(\mathrm{n}=2157)$ e primeiro seguimento $(\mathrm{n}=1709)$, de acordo com o município. Coorte SaMi-Trop. Minas Gerais - MG - Brasil.

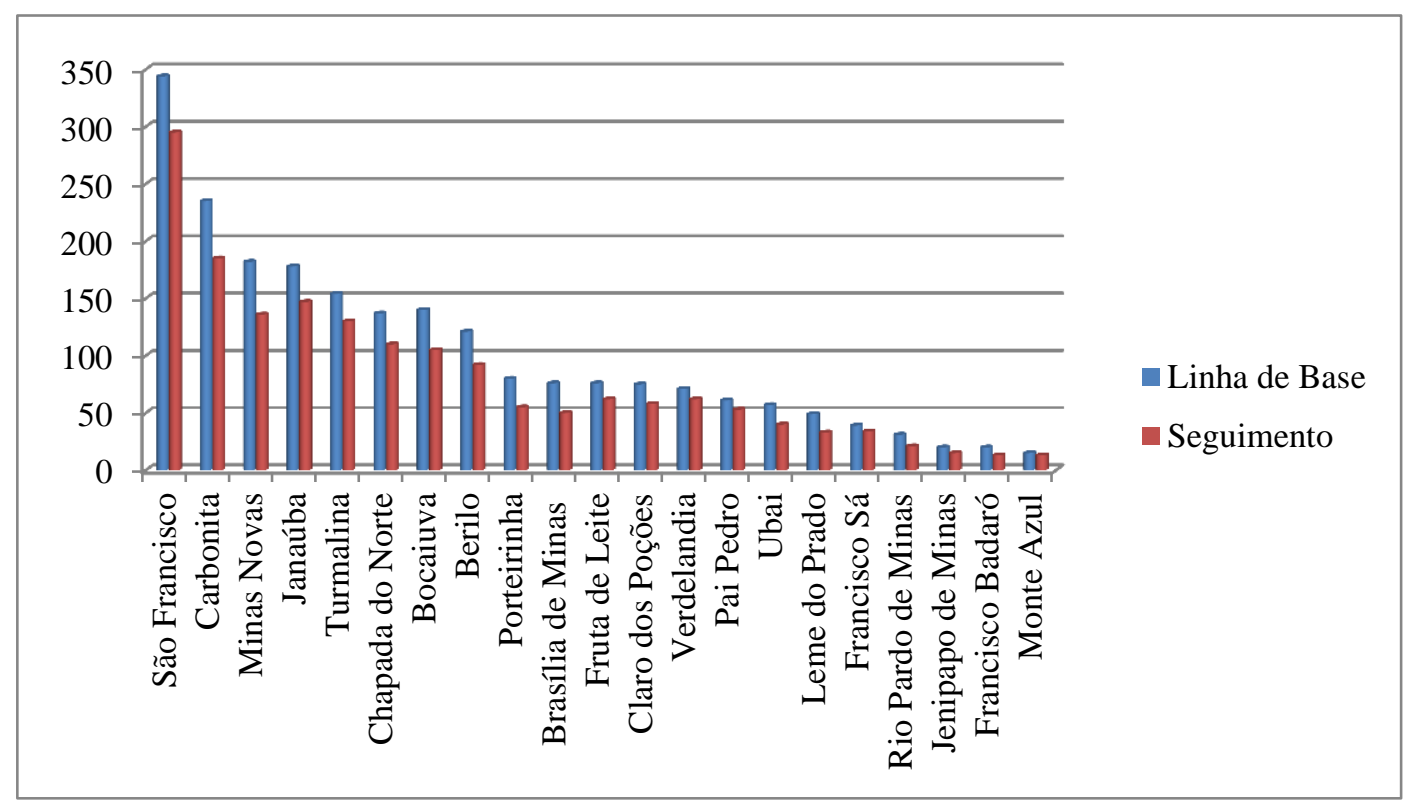


As principais características sociodemográficas, observadas na linha de base, são apresentadas na Tabela 1. A maioria dos participantes era do sexo feminino, apresentava idade até 60 anos, união estável, se autodeclarou não branco e alfabetizado. A renda per capta média era de $\mathrm{R} \$ 356,33$ (o salário mínimo vigente a época da coleta 2013-2014 - $\mathrm{R} \$ 724,00$ ). A maioria relatou apresentar hipertensão arterial, ter parentes portadores de $\mathrm{DC}$, não ter feito uso prévio do BZN, mas avaliou positivamente sua saúde. Quanto ao comportamento em saúde, a maioria declarou não praticar atividade física, não fazer uso frequente de álcool e não fumar (Tabela 1).

Tabela 1. Distribuição dos participantes da coorte, segundo características sociodemográficas, histórico médico e comportamento em saúde. Linha de base da coorte SaMi-Trop $(n=2157)$. Minas Gerais - MG - Brasil.

\begin{tabular}{|c|c|c|}
\hline \multirow[t]{2}{*}{ Características } & \multicolumn{2}{|l|}{ Descritivas } \\
\hline & $\mathbf{n}$ & $\%$ \\
\hline \multicolumn{3}{|l|}{ Características sociodemográficas } \\
\hline \multicolumn{3}{|l|}{ Sexo } \\
\hline Masculino & 716 & $33,2 \%$ \\
\hline Feminino & 1441 & $66,8 \%$ \\
\hline \multicolumn{3}{|l|}{ Idade } \\
\hline Até 60 anos & 1145 & $53,1 \%$ \\
\hline 60 anos ou mais & 1012 & $46,9 \%$ \\
\hline \multicolumn{3}{|l|}{ Renda per capita* } \\
\hline Superior à média percapita & 865 & $40,5 \%$ \\
\hline Inferior à média percapita & 1268 & $59,5 \%$ \\
\hline \multicolumn{3}{|l|}{ Estado Civil* } \\
\hline Com união estável & 1355 & $63,2 \%$ \\
\hline Sem união estável & 789 & $36,8 \%$ \\
\hline \multicolumn{3}{|l|}{ Cor da pele autodeclarada* } \\
\hline Branca & 479 & $22,4 \%$ \\
\hline Amarelo/indigena/pardo/preta & 1663 & $77,6 \%$ \\
\hline \multicolumn{3}{|l|}{ Alfabetizado* } \\
\hline $\operatorname{Sim}$ & 1180 & $54,7 \%$ \\
\hline Não & 964 & $44,7 \%$ \\
\hline \multicolumn{3}{|l|}{ Histórico médico } \\
\hline \multicolumn{3}{|l|}{ Colesterol alto* } \\
\hline Não & 1189 & $57,5 \%$ \\
\hline Sim & 880 & $42,5 \%$ \\
\hline
\end{tabular}


Hipertensão arterial*

Não

Autopercepção da saúde*

Positiva

Negativa

Qualidade de vida*

Insatisfatória

$7,8 \%$

Regular

Satisfatória

Doença de Chagas na família*

Sim

Uso prévio de BZN*

Sim

Portador de marca-passo*

Não

Sim

Classe Funcional*

Sem limitações

Com limitações

NTpro-BNP

Não alterado

Alterado

\section{Comportamento em saúde}

Prática de atividade física*

Não

Sim

Etilismo*

Uso não frequente de álcool $2090 \quad 97,7 \%$

Uso frequente de álcool

Tabagismo*

Nunca fumou ou ex-fumante

1978

$92,4 \%$

Fumante

162

$7,6 \%$

Entre a linha de base e o primeiro seguimento foi observado agravamento das condições cardíacas de alguns participantes: 45 (2,63\%) colocaram marca-passo, em 30 $(1,75 \%)$ surgiram fibrilação atrial, $155(9,1 \%)$ passaram a apresentar alteração do NT-proBNP e $244(14,3 \%)$ relataram ocorrência de hospitalização nos últimos dois anos. Condições 
relacionadas ao histórico médico como hipertensão arterial e infarto agudo do miocárdio sofreram variações de menos de $1 \%$ entre os dois períodos de observação. Já diabetes mellitus e colesterol alto variaram em $1,4 \%$ e $6,6 \%$, respectivamente. Houve aumento na autopercepção negativa da saúde de 14,5\% para 22,0\%. Prática de atividade física, etilismo e tabagismo também apresentaram variações de menos de $1 \%$.

Dentre os 1709 participantes incluídos no primeiro seguimento, observou-se que o serviço de saúde público era o principal utilizado, somente $1 \%(\mathrm{n}=21)$ apresentava plano de saúde. No que se refere ao acompanhados por médicos da ESF, 44\% relatou não terem este acompanhamento, 31\% morava a uma distância superior a $100 \mathrm{~km}$ da unidade de saúde mais próxima e $46 \%$ relatou pouca compreensão sobre sua condição de portador de DC (Tabela 2).

Tabela 2. Distribuição dos participantes, segundo o uso dos serviços de saúde. Primeiro seguimento da coorte SaMi-Trop $\left(n=1709^{¥}\right)$. Minas Gerais - MG - Brasil.

\begin{tabular}{|c|c|c|}
\hline \multirow[t]{2}{*}{ Características } & \multicolumn{2}{|l|}{ Descritivas } \\
\hline & $\mathbf{n}$ & $(\%)$ \\
\hline \multicolumn{3}{|l|}{ Usos dos serviços de saúde } \\
\hline \multicolumn{3}{|l|}{ Serviço de saúde para DC* } \\
\hline Público & 1126 & $67,0 \%$ \\
\hline Privado/ Plano de saúde & 391 & $23,3 \%$ \\
\hline Nenhum & 161 & $9,7 \%$ \\
\hline \multicolumn{3}{|l|}{ Residência acompanhada pela ESF* } \\
\hline Não & 1453 & $86,5 \%$ \\
\hline Sim & 169 & $10,1 \%$ \\
\hline Não sabe & 58 & $3,4 \%$ \\
\hline \multicolumn{3}{|l|}{ Acompanhamento pelo médico da ESF* } \\
\hline Sem acompanhamento & 734 & $43,7 \%$ \\
\hline Irregular & 476 & $28,3 \%$ \\
\hline Regular & 391 & $23,3 \%$ \\
\hline Não sabe & 79 & $4,7 \%$ \\
\hline \multicolumn{3}{|l|}{ Acompanhamento por médico especialista* } \\
\hline Sem acompanhamento & 993 & $59,1 \%$ \\
\hline Irregular & 324 & $19,3 \%$ \\
\hline Regular & 269 & $16,0 \%$ \\
\hline Não sabe & 94 & $5,6 \%$ \\
\hline \multicolumn{3}{|l|}{ Satisfação com o tratamento para DC* } \\
\hline Satisfeito/razoavelmente satisfeito & 800 & $47,6 \%$ \\
\hline
\end{tabular}


Insatisfeito/muito insatisfeito

Não sabe

Tempo desde a última consulta para DC*

Menos de um ano

Mais de um ano

Distância da residência à ESF*

Até $5 \mathrm{~km}$

De 6 a $99 \mathrm{~km}$

Acima de 100 km

Compreensão da condição de saúde para DC*

Compreende bem/ razoavelmente

Pouca compreensão

Não sabe

Medicamento gratuito para DC*

Sempre/ Frequentemente

Regularmente

Raramente/ Nunca

Não sabe

Frequências de exames para DC*

Sempre/ Frequentemente

Regularmente

Raramente/ Nunca

Variação do $n$ da linha de base de 2157 , devido a óbito $(n=146)$ e a perda de seguimento $(n=302)$ resultando em $n=1709$. *Variação no n de 1709 devido à perda de informações.

A descrição dos indivíduos com DC, segundo a ocorrência ou não de óbito em dois anos de seguimento, demonstrou que, dentre as 19 variáveis independentes consideradas, 13 se mostraram associadas ao óbito no nível de significância de 5 \% (Tabela 3).

Tabela 3 - Distribuição dos participantes, segundo a ocorrência ou não de óbito nos dois anos de seguimento. Primeiro seguimento da coorte SaMi-Trop, não óbito $(\mathrm{n}=1709)$, óbitos $(\mathrm{n}=$ 146). Minas Gerais - MG - Brasil.

\begin{tabular}{|c|c|c|c|c|c|}
\hline \multirow{2}{*}{ Características } & \multicolumn{2}{|c|}{ Não óbito } & \multicolumn{2}{|c|}{ Óbito } & \multirow{2}{*}{ P valor } \\
\hline & $\mathbf{n}$ & $\%$ & $\mathbf{n}$ & $\%$ & \\
\hline \multicolumn{6}{|l|}{ Características sociodemográficas } \\
\hline \multicolumn{6}{|l|}{ Sexo } \\
\hline Masculino & 557 & $89,8 \%$ & 63 & $10,2 \%$ & \multirow{2}{*}{0,009} \\
\hline Feminino & 1152 & $93,3 \%$ & 83 & $6,7 \%$ & \\
\hline \multicolumn{6}{|l|}{ Idade } \\
\hline Até 60 anos & 950 & $94,9 \%$ & 51 & $5,1 \%$ & \multirow{2}{*}{$\mathbf{0 , 0 0 0}$} \\
\hline 60 anos ou mais & 759 & $88,9 \%$ & 95 & $11,1 \%$ & \\
\hline \multicolumn{6}{|l|}{ Renda per capita* } \\
\hline Superior à média percapita & 675 & $90,8 \%$ & 68 & $9,2 \%$ & \multirow{2}{*}{$\mathbf{0 , 0 5 1}$} \\
\hline Inferior à média percapita & 1019 & $93,3 \%$ & 73 & $6,7 \%$ & \\
\hline \multicolumn{6}{|l|}{ Estado Civil* } \\
\hline Com união estável & 1106 & $93,2 \%$ & 81 & $6,8 \%$ & \multirow{2}{*}{0,048} \\
\hline Sem união estável & 598 & $90,6 \%$ & 62 & $9,4 \%$ & \\
\hline Cor da pele autodeclarada* & & & & & \\
\hline
\end{tabular}




\begin{tabular}{|c|c|c|c|c|c|}
\hline Branca & 375 & $91,9 \%$ & 33 & $8,1 \%$ & \multirow{2}{*}{0,739} \\
\hline Amarelo/indígena/pardo/preta & 1327 & $92,4 \%$ & 109 & $7,6 \%$ & \\
\hline \multicolumn{6}{|l|}{ Alfabetizado* } \\
\hline Sim & 953 & $94,4 \%$ & 57 & $5,6 \%$ & \multirow{2}{*}{$\mathbf{0 , 0 0 0}$} \\
\hline Não & 750 & $89,7 \%$ & 86 & $10,3 \%$ & \\
\hline \multicolumn{6}{|l|}{ Histórico Médico } \\
\hline \multicolumn{6}{|l|}{ Colesterol alto* } \\
\hline Não & 938 & $91,4 \%$ & 88 & $8,6 \%$ & \multirow{2}{*}{0,125} \\
\hline Sim & 706 & $93.4 \%$ & 50 & $6.6 \%$ & \\
\hline \multicolumn{6}{|l|}{ Diabetes Mellitus* } \\
\hline Não & 1524 & $92,1 \%$ & 131 & $7,9 \%$ & \multirow{2}{*}{0,662} \\
\hline Sim & 185 & $93,0 \%$ & 14 & $7,0 \%$ & \\
\hline \multicolumn{6}{|l|}{ Hipertensão arterial* } \\
\hline Não & 604 & $94,1 \%$ & 38 & $5,9 \%$ & \multirow{2}{*}{$\mathbf{0 , 0 2 6}$} \\
\hline Sim & 1105 & $91,2 \%$ & 107 & $8,8 \%$ & \\
\hline \multicolumn{6}{|l|}{ Doença de Chagas na família* } \\
\hline Não & 396 & $92,3 \%$ & 33 & $7,7 \%$ & \multirow{2}{*}{0,000} \\
\hline Sim & 1420 & $94,4 \%$ & 85 & $5,6 \%$ & \\
\hline \multicolumn{6}{|l|}{ Uso prévio de BZN* } \\
\hline Não & 1152 & $91,3 \%$ & 110 & $8,7 \%$ & \multirow{2}{*}{0,000} \\
\hline Sim & 430 & $96,8 \%$ & 14 & $3,2 \%$ & \\
\hline \multicolumn{6}{|l|}{ Portador de marca-passo* } \\
\hline Não & 1867 & $93,8 \%$ & 123 & $6,2 \%$ & \multirow{2}{*}{$\mathbf{0 , 0 0 0}$} \\
\hline Sim & 104 & $83,2 \%$ & 21 & $16,8 \%$ & \\
\hline \multicolumn{6}{|l|}{ Classe Funcional* } \\
\hline Sem limitações & 1079 & $95,7 \%$ & 48 & $4,3 \%$ & \multirow{2}{*}{$\mathbf{0 , 0 0 0}$} \\
\hline Com limitações & 916 & $81 \%$ & 95 & $19 \%$ & \\
\hline \multicolumn{6}{|l|}{ NTpro-BNP* } \\
\hline Não alterado & 1453 & $98,0 \%$ & 29 & $2,0 \%$ & \multirow{2}{*}{$\mathbf{0 , 0 0 0}$} \\
\hline Alterado & 511 & $81,9 \%$ & 113 & $18,1 \%$ & \\
\hline \multicolumn{6}{|l|}{ Autopercepção da saúde* } \\
\hline Positiva & 1455 & $92,4 \%$ & 120 & $7,6 \%$ & \multirow{2}{*}{0,483} \\
\hline Negativa & 236 & $91,1 \%$ & 23 & $8,9 \%$ & \\
\hline \multicolumn{6}{|l|}{ Qualidade de vida* } \\
\hline Insatisfatória & 83 & $90,8 \%$ & 9 & $9,2 \%$ & \\
\hline Regular & 501 & $96.2 \%$ & 20 & $3.8 \%$ & 0,125 \\
\hline Satisfatória & 528 & $92,8 \%$ & 35 & $7,2 \%$ & \\
\hline Comportamento em saúde & & & & & \\
\hline Prática de atividade física* & & & & & \\
\hline Não & 1293 & $91.3 \%$ & 123 & $8.7 \%$ & 0,018 \\
\hline Sim & 404 & $94,8 \%$ & 22 & $5,2 \%$ & 0,018 \\
\hline Etilismo* & & & & & \\
\hline Uso não frequente de álcool & 1577 & $91,8 \%$ & 140 & $8,2 \%$ & 0044 \\
\hline Uso frequente de álcool & 122 & $96,8 \%$ & 4 & $3,2 \%$ & 0,044 \\
\hline Tabagismo* & & & & & \\
\hline Nunca fumou ou ex-fumante & 1581 & $92,4 \%$ & 130 & $7,6 \%$ & 0.236 \\
\hline Fumante & 120 & $89,6 \%$ & 14 & $10,4 \%$ & 0,230 \\
\hline
\end{tabular}

* Variação no n de 1709 (primeiro seguimento), devido à perda de informações.

\section{DISCUSSÃO}

O projeto SaMi-Trop vem sendo desenvolvido e trazendo à tona uma realidade muitas vezes negligenciada, associada a DC, como o difícil acesso ao serviço de saúde e a falta de conhecimento e/ou atualização sobre a doença por parte dos profissionais de saúde. A linha de base desta coorte incluiu um total de 2157 participantes, sendo a maioria do sexo feminino, com idade até 60 anos, baixa renda per capta e com baixa escolaridade. No primeiro seguimento, dois anos após a linha de base, foram incluídos 1709 participantes. Houve perda de seguimento de 302 (14\%) participantes e $146(6,7 \%)$ faleceram. 
Entre a linha de base e o primeiro seguimento, observou-se que 2,6\% colocaram o marca-passo, em 1,75\% surgiram fibrilação atrial, 9,1\% passaram a ter alteração do NTproBNP, $14,3 \%$ relataram hospitalização no período, com consequente aumento da autopercepção negativa da saúde. Houve aumento de 6,6\% na prevalência de participantes com colesterol alto. O serviço de saúde utilizado, pela maioria dos participantes, foi o público, $43 \%$ relataram não receber acompanhamento por médicos da ESF, $30 \%$ moravam a uma distância superior a $100 \mathrm{~km}$ da unidade de saúde mais próxima e $46 \%$ relataram pouca compreensão sobre sua condição de portador de DC. De forma geral, o óbito esteve associado a piores condições sociodemográficas, histórico médico e comportamento em saúde.

O predomínio do sexo feminino em portadores de DC já foi evidenciado em estudos prévios ${ }^{15}, 16$. Mulheres utilizam os serviços de saúde com mais frequência, o que sugere maior facilidade no diagnóstico e manejo da $\mathrm{DC}^{17}$, enquanto homens, apresentam maior mortalidade por $\mathrm{DC}^{16}$. A idade média verificada entre os participantes do SaMi-Trop foi 59,6 ( $\pm 13,09)$ anos (dados não mostrados). O envelhecimento da população com DC já foi relatado $^{16,18}$, e representa um desafio para a assistência em virtude da associação e interação com outras comorbidades, como diabetes mellitus e hipertensão ${ }^{18}$. Foi também verificado baixo perfil econômico dos portadores de DC (maioria com renda per capta inferior a $\mathrm{R} \$ 356,33)$, estando abaixo do valor da renda per capta média do estado de Minas Gerais MG $(\mathrm{R} \$ 733,24)^{19}$. A taxa de analfabetismo verificada entre os participantes do SaMi-Trop $(44,7 \%)$ também foi bastante superior as taxas verificadas para a população de Minas Gerais MG $(8,1 \%)$ e do Brasil $(9,4 \%)$. As regiões do Vale do Jequitinhonha e do Norte de Minas Gerais - MG, representam as segundas e terceiras regiões com a maior taxa de analfabetos no estado (17,6\% e 15,8\%, respectivamente). Ainda assim, a prevalência de analfabetismo entre os portadores de DC foi mais que o dobro da verificada na região ${ }^{20,21}$. De forma geral, o perfil sociodemográfico observado foi coerente com populações portadoras de $\mathrm{DC}^{22}$ e com estudos locais $^{20}$.

O óbito, assim como a fibrilação atrial e implantação de marca-passo, representa, de forma nítida, o agravamento das condições cardíacas entre portadores de $\mathrm{DC}^{23}$. No decorrer de dois anos de seguimento, foi registrado cerca de 7\% de óbitos entre os participantes da coorte SaMi-Trop em virtude do agravamento de suas condições cardíacas. Este é um dado alarmante, principalmente ao se considerar que dentre os indivíduos com DC, 
$20 \%$ a $40 \%$ apresentam alterações cardíacas $^{16}$. Também, neste período, $1,75 \%$ dos participantes passaram a apresentar fibrilação atrial, uma anormalidade do ECG típica da CCC, indicativa de pior prognóstico por estar associada ao maior risco de mortalidade ${ }^{24}$. Observou-se, também, que cerca de $9 \%$ dos participantes passaram a apresentar alteração dos níveis de NT-proBNP em dois anos. O NT-proBNP já foi reconhecido como o fator que mais fortemente se associou a eventos cardiovasculares ${ }^{4}$, sendo um discriminador preciso do diagnóstico de insuficiência cardíaca e preditor importante de óbito ${ }^{24}$. O implante de marcapasso é o desfecho cardíaco mais importante da DC, que ocorre em consequência da $\mathrm{CCC}^{4,24}$. Na coorte SaMi-Trop, foi observada uma incidência de 2,63\% de implante de marca-passo nos últimos dois anos. A DC, ainda, é uma das principais causas de implante de marca-passos no Brasil ${ }^{4,25}$, representando um risco treze vezes maior quando comparado com outras causas $^{23}$. A coorte SaMi-Trop observou taxa de $14,3 \%$ de hospitalização entre portadores de DC em um período de 2 anos. Estudo conduzido entre portadores de DC encontrou taxa de hospitalização de 33\% ao ano no estado de Minas Gerais - MG, na década de 90. Os autores verificaram que as taxas de hospitalização haviam reduzido em relação aos anos anteriores ${ }^{26}$.

A maioria dos portadores de DC da coorte SaMi-Trop relataram utilizar os serviços públicos de saúde $67 \%$, embora $43,7 \%$ não estejam recebendo acompanhamento por médicos da ESF na condução da DC. Permeando as questões de acesso ao serviço de saúde, há que se destacar a dificuldade que muitos portadores de DC enfrentam no deslocamento até as unidades de saúde. Foi observado que 30\% dos portadores de DC residiam a uma distância superior a $100 \mathrm{~km}$ da unidade de saúde mais próxima. Além disso, estudo prévio verificou que nos municípios participantes desta coorte, há uma média de 0,68 $( \pm 0,4)$ médicos por mil habitantes $^{23}$, sendo quase três vezes menor que a média nacional $(5,07)$ e metade da média observada entre municípios do interior do Brasil $(1,28)^{27}$, o que confirma se tratar de uma região carente economicamente e com pouca estrutura em saúde ${ }^{23}$.

Chamou atenção a falta de compreensão sobre o estado de saúde do portador de DC, relatada por quase metade, 46,2\%, dos participantes da coorte SaMi-Trop. A falta de compreensão sobre a doença implica em um prejuízo no curso do tratamento da DC, podendo contribuir com seu agravamento $^{28}$. Além disso, observou-se $14,5 \%$ dos portadores de DC com autopercepção negativa de sua saúde. A autopercepção é um importante preditor de 
mortalidade, preditor do declínio funcional, além de influenciar a procura por atendimento em saúde $^{28}$.

O óbito decorrente da DC esteve associado a piores condições sociodemográficas e clínicas, concordando com estudos prévios. A idade acima de $60 \operatorname{anos}^{4,}$ 29, o sexo masculino $^{29}$, baixa renda, baixa escolaridade ${ }^{30}$, a ausência de união estável ${ }^{16}$ e a presença de DC na família ${ }^{31}$ foram associados a maior mortalidade na DC. As variáveis do histórico médico que apresentaram associação com o óbito foram presença de hipertensão arterial, não uso prévio de BZN, ser portador de marca-passo, classe funcional com limitação e NTproBNP alterado. Tais eventos estão relacionados ao possível agravamento da DC e ao dano cardíaco $^{32}$, conforme já discutido. A classe funcional categoriza a extensão da insuficiência cardíaca, sendo que indivíduos sem limitação não apresentam restrição funcional em suas atividades rotineiras ${ }^{33}$. A associação da classe funcional com o pior prognóstico da DC também já foi reconhecida ${ }^{32}$. O BZN é o único medicamento antiparasitário que age no $T$. cruzi, permitido para comercialização no Brasil ${ }^{34}$. Estudos prévios verificaram que o uso do BZN esteve associado a uma redução significativa da parasitemia ${ }^{32}$, menor prevalência de marcadores de cardiomiopatia grave e menor mortalidade, enquanto o não uso do BZN aumentou a chance de eventos cardiovasculares ${ }^{13}$. Sendo assim, os achados da coorte SaMiTrop, também, evidenciam que a gravidade clínica do portador de DC é associada ao óbito ${ }^{35}$.

A perda de seguimento de $14 \%$ dos participantes em um período de dois anos está coerente com estudos de acompanhamento longitudinal ${ }^{36}$. Uma revisão sistemática de estudos de coorte evidenciou a permanência de $73,5 \%$ dos participantes em uma média de 4,3 anos ${ }^{36}$. A validade da amostra no primeiro seguimento da coorte SaMi-Trop foi considerada em estudo prévio. Ao se comparar o perfil sociodemográfico dos indivíduos que permaneceram, com aqueles que foram perdidos durante o primeiro seguimento do estudo, foi verificado que não havia diferenças significativas para a maioria das variáveis.

Além da perda de segmento, inerente a estudos longitudinais, pode-se mencionar como limitação da coorte SaMi-Trop o fato de algumas características clínicas, tais como: diagnóstico de hipertensão, diabetes, uso do BZN, entre outros, terem sido aferidas através de autorrelato, não podendo desconsiderar a possibilidade de viés. Por outro lado, a coorte SaMiTrop é a maior e mais longa coorte de acompanhamento de pacientes com DC que se tem notícia atualmente. Esta coorte tem apresentado resultados relevantes, uma vez que já 
identificou taxas de mortalidade de acordo com o risco ${ }^{12}$, o perfil de usuários do $\mathrm{BZN}^{13}$, forneceu informações relevantes sobre o desenvolvimento e progressão da DC, marcadores clínicos e laboratoriais ${ }^{13,23}$, dentre outras importantes publicações científicas acerca da DC.

\section{CONCLUSÃO}

A coorte SaMi-Trop é um estudo multicêntrico que vem acompanhando aproximadamente 2000 portadores de DC de regiões endêmicas do estado de Minas Gerais MG. Uma amostra robusta, rica em informações, que teve início nos anos de 2013 e 2014, e, posteriormente, um primeiro seguimento nos anos de 2015 a 2016. Um novo seguimento de avaliação está em andamento, tendo sido iniciado no ano de 2019. A coorte SaMi-Trop tem apresentado informações relevantes que contribuem para o avanço no conhecimento científico da DC. Seus resultados também devem subsidiar políticas públicas e estratégias para melhorar os serviços de saúde ofertados, de forma direcionada, às reais necessidades dos portadores de DC no norte de Minas Gerais.

\section{REFERÊNCIAS}

1. DIAS, João Carlos Pinto et al. II Consenso Brasileiro em Doença de Chagas, 2015. Epidemiol. Serv. Saúde, Brasília, v. 25, n. esp, p. 7-86, jun. 2016.

2. LIMA, Ronildo de Souza; TEIXEIRA, Andrea Bessa; LIMA, Vera Lucia da Silva. Doença de Chagas: uma atualização bibliográfica. RBAC, v. 51, n. 2, p. 103-06, 2019.

3. ANGHEBEN, Andrea et al. Rapid immunochromatographic tests for the diagnosis of chronic Chagas disease in at-risk populations: A systematic review and meta-analysis. PLoS neglected tropical diseases, v. 13, n. 5, p. e0007271, 2019.

4. NUNES, Maria Carmo Pereira et al. Chagas Cardiomyopathy: An Update of Current Clinical knowledge and Management: A Scientific Statement From the American Heart Association. Circulation, v. 138, n. 12, p. e169-e209, 2018.

5. PEREIRA, Cláudia Marta Luiz et al. Perfil clínico e epidemiológico da doença de chagas aguda no estado de Minas Gerais. Revista de Atenção à Saúde, v. 15, n. 52, p. 49-54, 2017. 
6. LIMA, Neiberg de Alcantara et al. Preditores de mortalidade em pacientes com cardiopatia isquêmica e cardiopatia chagásica crônica com cardiodesfibrilador implantável. Journal Of CardiacArrhythmias, v. 29, n. 3, p. 95-100, 2016.

7. RASSI JR, Anis; MARIN, José Antonio; RASSI, Anis. Chronic Chagas cardiomyopathy: a review of the main pathogenic mechanisms and the efficacy of aetiological treatment following the BENznidazole Evaluation for Interrupting Trypanosomiasis (BENEFIT) trial. Memórias do Instituto Oswaldo Cruz, v. 112, n. 3, p. 224-235, 2017.

8. FERREIRA, Ariela Mota et al. Avaliação do Conhecimento Acerca do Manejo Clínico de Portadores da Doença de Chagas em Região Endêmica no Brasil. Revista de APS, v. 21, n. 3, 2018.

9. MELO, Tatiana G et al. Trypanosoma cruzi down-regulates mechanosensitive proteins in cardiomyocytes. Memórias do Instituto Oswaldo Cruz, v. 114, 2019.

10. SIMÕES, Marcus Vinicius et al. Cardiomiopatia da doença de Chagas. International Journal of Cardiovascular Sciences, v. 31, n. 2, p. 173-189, 2018.

11. CARDOSO, Clareci Silva et al. Longitudinal study of patients with chronic Chagas cardiomyopathy in Brazil (SaMi-Trop project): a cohort profile. BMJ open, v. 6, n. 5, 2016.

12. OLIVEIRA, Claudia Di LORENZO et al. Risk Score for Predicting 2-Year Mortality in Patients With Chagas Cardiomyopathy From Endemic Areas: SaMi-Trop Cohort Study. Journal of the American Heart Association, v. 9, n. 6, p. e014176, 2020.

13. CARDOSO, Clareci Silva et al. Longitudinal study of patients with chronic Chagas cardiomyopathy in Brazil (SaMi-Trop project): a cohort profile. BMJ open, v. 6, n. 5, 2016.

14. ALKMIM, Maria Beatriz et al. Improving patient access to specialized health care: the Telehealth Network of Minas Gerais, Brazil. Bulletin of the World Health Organization, v. 90, p. 373-378, 2012.

15. MARTINS-MELO, Francisco Rogerlândio et al. Prevalence of Chagas disease in Brazil: A systematic review and meta-analysis. Acta Tropica, v. 130, p. 167-174, 2014. 
16. DA NÓBREGA, Aglaêr Alves; de ARAUJO, Wildo Navegantes; VASCONCELOS, Ana Maria Nogales. Mortality Due to Chagas disease in Brazil according to a specific cause. The American journal of tropical medicine and hygiene, v. 91, n. 3, p. 528-533, 2014.

17. MALTA, Deborah Carvalho et al. Social inequalities in the prevalence of selfreported chronic non-communicable diseases in Brazil: national health survey 2013. International Journal for Equity in Health, v. 15, n. 1, p. 153, 2016.

18. LIMA-COSTA, Maria Fernanda; VERAS, Renato. Saúde pública e envelhecimento. Cadernos de Saúde Pública, v. 19, n. 3, p. 700-701, 2003.

19. INSTITUTO BRASILEIRO DE GEOGRAFIA E ESTATÍSTICA (IBGE) Censo Demográfico 2010: demográficas e socioeconômicas.

20. PÉREZ-MOLINA, José A.; MOLINA, Israel. Chagas disease. The Lancet, v. 391, n. 10115, p. 82-94, 2018.

21. PEREIRA, José Borges; WILLCOX, Henry Percy; COURA, José Rodrigues. Morbidade da doença de Chagas: III. Estudo longitudinal, de seis anos, em Virgem da Lapa, MG, Brasil. Memórias do Instituto Oswaldo Cruz, v. 80, n. 1, p. 63-71, 1985.

22. MARTINEZ, Felipe et al. Chagas disease and heart failure: an expanding issue worldwide. European Cardiology Review, v. 14, n. 2, p. 82, 2019.

23. FERREIRA, Ariela Mota et al. Impact of the social context on the prognosis of Chagas disease patients: Multilevel analysis of a Brazilian cohort. PLoS neglected tropical diseases, v. 14, n. 6, p. e0008399, 2020.

24. RIBEIRO, Antonio Luiz P. et al. Electrocardiographic abnormalities in elderly Chagas disease patients: 10-year follow-up of the Bambui Cohort Study of Aging. Journal of the American Heart Association, v. 3, n. 1, p. e000632, 2014.

25. MOND, Harry G.; PROCLEMER, Alessandro. The 11th world survey of cardiac pacing and implantable cardioverter-defibrillators: calendar year 2009-a World Society of Arrhythmia's project. Pacing and clinical electrophysiology, v. 34, n. 8, p. 1013-1027, 2011.

26. FRANÇA, Susete Barbosa; de ABREU, Daisy Maria Xavier. Morbidade hospitalar por doença de Chagas no Brasil. Revista da Sociedade Brasileira de Medicina Tropical, v. 29, n. 2, p. 109-115, 1996. 
27. SCHEFFER, Mario; BIANCARELLI, Aureliano; CASSENOTE, Alex. Demografia médica no Brasil 2015. Departamento de Medicina Preventiva, Faculdade de Medicina da USP, São Paulo p. 284, 2018.

28. CAVALCANTI, Marília Abrantes Fernandes et al. Manifestações e estratégias de enfrentamento da Doença de Chagas que interferem na qualidade de vida do indivíduo: uma revisão sistemática. Ciência \& Saúde Coletiva, v. 24, p. 1405-1416, 2019.

29. IDLER, E.L.; BENYAMINI, Y. Self-rated health and mortality: a review of twentyseven community studies. Journal of health and social behavior, v.38, n. 1, p. 21-37, 1997.

30. CAMM, A John et al. 2012 focused update of the ESC Guidelines for the management of atrial fibrillation: an update of the 2010 ESC Guidelines for the management of atrial fibrillation. Developed with the special contribution of the European Heart Rhythm Association. Eur Heart J, v. 33, n. 21, p. 2719-2747, 2012.

31. CUCUNUBÁ, Zulma M. et al. Increased mortality attributed to Chagas disease: a systematic review and meta-analysis. Parasites \& vectors, v. 9, n. 1, p. 42, 2016.

32. FERREIRA, Ariela Mota et al. Benznidazole Use among Patients with Chronic Chagas' Cardiomyopathy in an Endemic Region of Brazil. Plos one, v. 11, n. 11, p. e0165950, 2016.

33. CHACKO, K. A. AHA Medical/Scientific Statement: 1994 revisions to classification of functional capacity and objective assessment of patients with diseases of the heart. Circulation, v. 92, n. 7, p. 2003-2005, 1995.

34. MINISTÉRIO DA SAÚDE. Secretaria de Vigilância em Saúde. [Consenso Brasileiro de Doença de Chagas]. Revista da Sociedade Brasileira de Medicina Tropical. 2005; 38 Suplemento 3: 7-29.

35. NUNES, Maria Carmo Pereira; FERRARI, Teresa Cristina Abreu. Mitral-Aortic Intervalvular Fibrosa: A Hidden Region Associated With Infective Endocarditis Complications. JACC Journals, v.2, n.8, p. 1217-1219, 2020.

36. TEAGUE, Samantha et al. Retention strategies in longitudinal cohort studies: a systematic review and meta-analysis. BMC medical research methodology, v. 18, n. 1, p. $151,2018$. 